\title{
Utilizing simulation curriculum to decentralize mental health concepts
}

\author{
D. M. H. Michelle Beckford
}

Saint Peter's University, Jersey City, NJ, USA

Email: mbeckford@saintpeters.edu

Received 14 March 2013; revised 15 April 2013; accepted 10 May 2013

Copyright @ 2013 D. M. H. Michelle Beckford. This is an open access article distributed under the Creative Commons Attribution License, which permits unrestricted use, distribution, and reproduction in any medium, provided the original work is properly cited.

\section{ABSTRACT}

The International Society of Psychiatric Nurses as well as The American Psychiatric Nurses Association called for decentralization of psychiatric concepts in undergraduate nursing education. The author developed a series of simulation scenarios to address therapeutic communication and psychosocial needs in a variety of patient care settings. In terms of holistic care, psychological well-being is relevant to all realms of nursing practice. Mental health assessment and intervention need to be included in all areas of an undergraduate-nursing curriculum. The purpose of this project was to create a BSN curriculum that reflects the mental health needs of patients not only in acute care psychiatric facilities but also in medicalsurgical disciplines, maternity units, and community settings. The curriculum is designed to also cover mental health throughout the lifespan-from infancy to end of life. A goal was to develop educational techniques, which translate into safe practice. A series of five simulations have been created with additional scenarios to be established in the following academic year. Topics include: alcohol withdrawal, end of life care, post-traumatic stress disorder, post-partum depression, serotonin syndrome, benzodiazepine over dose, elder abuse, depression in military veterans, and geriatric depression. All incorporate QSEN competencies [1]. The overall learning objectives are for undergraduate nursing students to initiate and implement an appropriate mental status assessment using therapeutic communication, and also to provide safe care by identifying risk factors and using clinical decision making to establish requisite nursing interventions. The methodology included relevant documentation through the use of appropriate assessment tools. Learning objectives were assessed through presimulation and post-simulation ten-question inventory and anecdotal feedback. QSEN competencies were utilized in creating scenarios as well as pre and post testing. Students voiced an ability to connect the didactic material to their clinical experiences following simulation.

Keywords: Simulation; Psychosocial; Communication

\section{INTRODUCTION}

Nurse educators are being challenged to evolve with the changing times; the needs of new graduates are rigorous and compelling. According to the Institute of Medicine: The Future of Nursing: Leading Change, Advancing Health-Report Briefing, 80\% of Registered Nurses (RNs) will be expected to enter practice with a Bachelor of Science in Nursing (BSN) by the year 2020 [2]. Confounded by an aging population in the US, the need for competent and proficient graduates has never been greater. The Health Resources and Services Administration (HRSA) projects that nursing schools will need to graduate $90 \%$ more RNs to meet the expected demand [3]. Traditional didactic classroom lectures may not suffice to meet the critical thinking needs that will be required of those entering practice within complex high acuity situations. The curriculum must extend beyond the didactic to facilitate psychomotor skills and application to clinical care of patients. The National Council on State Boards of Nursing [4] defines simulation as “... as a strategy—not a technology - to mirror, anticipate, or amplify real situations with guided experiences in a fully interactive way." McGuiness [5] called for innovative teaching strategies utilizing simulation with regard to psychiatric nursing. In the current climate schools of nursing struggle to keep pace with tight budgets, high acuity and short length of stays for hospitalized patients, and difficulty finding clinical sites for students. Simulation is an option that enhances clinical experiences and prepares nursing students for transition to safe practice. The author has developed a 
curriculum designed to decentralize mental health concepts, thereby emphasizing the need for psychosocial care and therapeutic communication in all realms of patient care. The curriculum addresses current trends in healthcare with components such as post-traumatic stress following sexual assault, elder abuse, and organ donation.

\section{OBJECTIVES}

The development of the MentalHealthSim curriculum began as a collaborative endeavor utilizing the skills of nurse educators with diverse practice experiences. The underlying premise was that mental health assessment and intervention needs to be threaded across all areas of the nursing curriculum. The purpose was to create a quality nursing curriculum that reflects the psychosocial needs of patients in acute psychiatric facilities, acute medical-surgical units, women's health out patient settings, community settings, pediatric intensive care unit, and child/adolescent services. Thus while the current trend in nursing education is de-emphasizing specialties such as maternal health and pediatrics, the authors have suggested curriculum which denotes the importance of psychosocial integrity as part of holistic care for all patients in all settings.

An initial approach to designing this curriculum approach was to include students in all levels of a Bachelor of Science program. Therefore the author planned for inclusion in the clinical courses for sophomores, juniors, and seniors. Therapeutic communication became the commonality from which to reinforce concepts learned as students progressed through the program. This strategy also served to promote the importance of establishing a trusting therapeutic relationship through the use of interpersonal skills as opposed to perpetuating a task-oriented approach. The scenarios are unique in that interactions are prompted by patient verbal and/or non-verbal behavior rather than by the traditional scenario that focuses on physiological parameters.

Perhaps the most innovative feature of MentalHealthSim is found within the content of each scenario. Each scenario created consists of patient care issues that are not widely represented in current literature. Topics include such content as care of the suicidal military veteran, post-traumatic stress symptoms status post sexual assault, end of life care and organ donation in an infant. Utilizing simulation in the lab setting allows the student to benefit and learn from these experiences which they may never encounter as a student but will likely encounter as a licensed clinician. Such scenarios are intended to promote caring and empathy, thus overcoming the notion that the use of technology has to be impersonal.

A series of five simulation scenarios have been created thus far as the substance for the curriculum design. These included alcohol withdrawal in the young adult, elder abuse in the community, post-traumatic stress disorder in adolescent outpatient care, depression in the military veteran, end of life and organ donation in the pediatric intensive care. Each scenario involved complex patient care needs based upon an underlying medical condition with subsequent changes in mental status. Students were prepared for impending scenarios through didactic lecture and assigned readings of relevant literature. The suspension of disbelief was enhance via the use of appropriate environmental props i.e. the elder patient in the community dressed in PJs, military vet wearing dog tags etc. Facilitators provided students with an intentionally brief introduction and patient history. Students were expected to perform appropriate assessments and to prioritize their interventions based upon the unfolding scenario. Debriefing allowed for students to review important concepts, to discuss questions and concerns, and to provide anecdotal feedback. Data was gathered via the use of a 10-question pre and posttest survey. As per QSEN competencies, each survey also included a dosage calculation requirement [6].

\section{OUTCOMES}

Therapeutic communication is a skill introduced to students during their first clinical course and reinforced through subsequent classes. However, students are rarely engaged in sensitive topics of discussion during actual clinical rotations. Students do not have their psychiatric nursing rotation until the fall semester of their senior year and therefore were not expected to diagnosis as part of the scenario but were expected to be able to communicate effectively, to provide safety measures, and to recognize the need for collaboration and/or need for referrals. In debriefing sessions, students voiced appropriate concerns about being caught off guard by patient concerns and not knowing how to respond. It is worth noting however that students in the junior class had back to back scenarios over two consecutive weeks and those students voiced improvement in their communication skills and were noticeably more responsive in problem solving with patient.

Students rated their ability to prioritize nursing interventions for safe patient care very strongly on pretests for all scenarios. Post-test scores in the alcohol withdrawal scenario reflected students' difficulty in identifying safety measures as evidenced by a decreased rating in terms of the self statement regarding ability to identify and prioritize three nursing interventions, Scores dropped roughly $20 \%$ from the pre-test to the post-test on this measure. Likewise, students rated themselves as not comfortable in providing holistic care to a patient with a spi- 
nal cord injury nor able to prioritize interventions for safe care with the same population. Lower scores on self assessments in the post-test may reflect the complexity of learning needs and further substantiates the need for teaching this content.

Anecdotally students identified an ability to transfer knowledge between simulations in the lab to patient care in the clinical setting. Students consistently have requested the opportunity to participate in more simulated scenarios.

\section{DISCUSSION}

MentalHealthSim is a new and unique educational tool for teaching holistic nursing care. The author has addressed a gap in the literature with regard to simulation in psychiatric nursing. While simulation, is not new in nursing, the scenarios created in this simulation have a psychosocial focus along with traditional physical care. The focus is on therapeutic communication and emotional well-being. This type of simulation enables students to address the patient in a more comprehensive manner.

Simulation is a valuable educational tool in that learning environment is a non-threatening place, where it is safe to make mistakes. Debriefing was utilized as a component to review mistakes and clarify student apprehensions. This curriculum has served to decrease student anxiety as they prepare for clinical rotations.

Simulation offers standardized clinical experiences for the purpose of teaching critical concepts that students are unlikely to encounter. It allows for additional patient care scenarios of varying acuity in an era when short-term care facilities have short length of stay. Simulation has the added benefit of debriefing, which allows for reflection and inquiry.

MentalHealthSim is useful in threading mental health concepts throughout the curriculum. It emphasizes the importance of psychosocial integrity while decentralizing therapeutic communication and psychosocial skills, thereby making them tools that are applicable in all patient care situations.

Future consideration will be given to developing additional scenarios related to mental health concepts. Scenarios may include shaken baby syndrome, tricyclic antidepressant overdose in the pregnant teen, and futile care with an ethics review board referral. In the future, stu- dents will be given the post-test at a six-month interval following simulation date to assess learning and retention. Future research may include the use of video taping to document verbal and nonverbal exchanges between student and patient. The author suggests building upon the research of Roberts and Bucksey [1] in utilizing the Medical Communications Behavior System to analyze verbal communication. Tracking standardized testing scores on psychosocial concepts may be another area of relevance for research. Finally, future plans will include not only collecting aggregate data from student surveys, but will include feedback from faculty in terms of rating student performance on learning objectives and expected outcomes.

\section{ACKNOWLEDGEMENTS}

A very special thanks to Patricia Ahearn, M.S.N., RN, Dr. Corinne Ellis, D.N.P., RN, BC MargaretMacali, RN, PHCNS-BC, Michelle Morales, M.S.N., RN-BC for their tireless support and expertise.

\section{REFERENCES}

[1] Roberts, L. and Bucksey, S.J. (2007) Communicating with patients: What happens in practice? Physical Therapy, 87, 586-594. doi:10.2522/ptj.20060077

[2] Institute of Medicine of the National Academies (2012) Advising the nation improving health. http://www.iom.edu/Reports/2010/The-Future-of-Nursing -Leading-Change-Advancing-Health/Report-Release.aspx

[3] Alden, K.R. (2008) Predictors of early academic success and program completion among baccalaureate nursing students. Graduate Faculty of North Carolina State University, Raleigh.

[4] National Council State Boards of Nursing (2007) The role of simulation in nursing education: A regulatory perspective.

https://www.ncsb.org/The_Role_of_Simulation_in_Nursi ng_Education

[5] McGuiness, T.M. (2011) Simulation in psychiatric nursing education. Journal of Psychosocial Nursing and Mental Health Services, 49, 9-10. doi:10.3928/02793695-20110329-05

[6] Sherwood, G. and Barnsteiner, J. (2012) Quality and safety in nursing: A competency approach to improving outcomes. John Wiley \& Sons, Hoboken. 